Pacific Journal of Mathematics

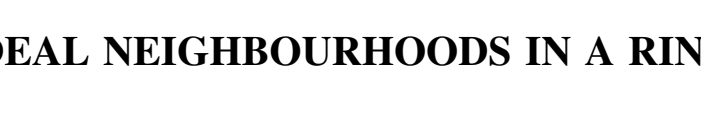




\title{
IDEAL NEIGHBOURHOODS IN A RING
}

\author{
H. SUbRAMANiAN
}

\begin{abstract}
A group topology on a ring is said to have ideal closure property in case the closure of an ideal is the intersection of all maximal ideals containing it. Hinrichs considered such group topologies on rings $C(X)$ of continuous real-valued functions defined over completely regular Hausdorff spaces. He gave a characterization of such topologies with ideal neighbourhoods at zero in $C(X)$, and showed that there exists in $C(X)$ a group topology with ideal closure property with the largest collection of open ideals. His results are indeed true in a wider class of rings-viz. semisimple commutative rings with unit element whose structure spaces of maximal ideals (with hull-kernel topology) are Hausdorff. This generalization is achieved by making use of a characterization by Gillman of such rings.
\end{abstract}

In this paper, a ring will always mean a semisimple commutative ring with unit. With every maximal ideal $M$ of a ring $R$, let us associate an ideal $\pi(M)$ consisting of all elements $x$ in $R$ such that $x$ belongs to all maximal ideals forming a certain neighbourhood of $M$ in the structure space $\mathfrak{M}(R)$ of maximal ideals of $R$ [1]. The ideal $\pi(M)$ can be algebraically characterized as the set $\{x \in R \mid$ there exists $y \notin M$ such that $x y=0\}$. It is known [1] that the structure space $\mathfrak{M}(R)$ of maximal ideals of $R$ is Hausdorff if and only if for every $M \in \mathfrak{M}(R), M$ is the unique maximal ideal containing $\pi(M)$. In what follows, a ring $R$ shall also be such that $\mathfrak{M}(R)$ is Hausdorff. We may mention that this class of rings includes all (von Neumann) regular rings, rings of all (bounded) uniformly continuous real-(complex-) valued functions defined over a uniform space, and $L^{1}(G)$ 's (with unit element adjointed whenever needed) for locally compact Abelian groups $G$.

By a group topology on a ring we mean the topology which makes the additive group structure of the ring a topological group (not necessarily Hausdorff). The ideal closure property of a group topology on a ring is written shortly as I.C.P. Obviously, the closed ideals in a ring with a group topology with I.C.P. are precisely the intersections of maximal ideals. We will make use of the well-known fact that any open subgroup of a topological group is closed.

THEOREM 1. Let $R$ be a ring with some group topology $\tau$. If $\tau$ has I.C.P., then $\mathrm{Cl} \pi(M)=M$ for every maximal ideal $M$ of $R$. The converse is true if further $\tau$ has an ideal basis of neighbourhoods at zero. 
Proof. We need prove only the converse since the first part is easy as $\mathfrak{M}(R)$ is assumed to be Hausdorff. Consider any ideal $I$, all of whose maximal divisors are $\left\{M_{\alpha}\right\}$. Let $V$ be any open ideal. Taking $x \in \cap M_{\alpha}$, we see that for each $\alpha$, there exists an element $a_{\alpha} \in V$ such that $x+a_{\alpha} \in \pi\left(M_{\alpha}\right)$. Therefore there exist elements $b_{\alpha} \notin M_{\alpha}$ such that $\left(x+a_{\alpha}\right) b_{\alpha}=0$. That is, $x b_{\alpha} \in V$. Since the ideal $\left(I, \cdots, b_{\alpha}, \cdots\right)$ cannot be contained in any maximal ideal of $R$, we will have some $i \in I$ such that $i+$ a finite (ring) linear combination of $b_{\alpha}$ 's equal to 1 . So, $x+$ an element of $V$ belongs to $I$. This means that, under the hypothesis of ideal basis of neighbourhoods at zero (an ideal neighbourhood of zero is necessarily an open ideal an conversely), $x$ belongs to $\mathrm{Cl} I$. As every maximal ideal is evidently closed, $\mathrm{Cl} I$ is contained in $\cap M_{\alpha}$; whence the desired result follows.

Lemma. Suppose that $I_{1}$ and $I_{2}$ are two ideals of a ring $R$ such that for some maximal ideal $M$ of $R$ and some $x \in M,\left\{x+I_{j}\right\} \cap \pi(M)$ is nonnull, $j=1,2$. Then $\left\{x+I_{1} \cap I_{2}\right\} \cap \pi(M)$ is nonnull.

Proof. We have $x+a_{j} \in \pi(M), a_{j} \in I_{j}, j=1,2$. So there exist $b j \in M, j=1,2$ such that $\left(x+a_{j}\right) b_{j}=0$, which gives that $x b_{1} b_{2} \in I_{1} \cap$ $I_{2}$. Since $\left(b_{1} b_{2}, \pi(M)\right)=R$, there exists an element $h \in R$ such that $h b_{1} b_{2}-1 \in \pi(M)$. Obviously then, $\left\{x+I_{1} \cap I_{2}\right\} \cap \pi(M)$ is nonnull.

THEOREM 2. The family of all maximal ideals of a ring $R$ defines a ring topology in $R$ having I.C.P. This is a Hausdorff topology whose open ideals are precisely the finite intersections of maximal ideals.

Proof. Any nonempty family of ideals in $R$ will define a ring topology on it, if they are considered as a subbase for the neighbourhood system at zero. The ring topology given by all maximal ideals is Hausdorff because the ring is semisimple.

In view of the preceding theorem and lemma, to see that this topology has I.C.P., it is sufficient to prove that, if $M_{1}$ and $M_{2}$ are any two maximal ideals and $x \in M_{1}$ then $\left\{x+M_{2}\right\} \cap \pi\left(M_{1}\right)$ is nonnull. If $M_{1}=M_{2}$, this is obvious; we take $-x \in M_{2}$. If $M_{1}$ and $M_{2}$ are distinct we have $\pi\left(M_{1}\right)+M_{2}=R$; hence, $a+b=1, a \in \pi\left(M_{1}\right), b \in M_{2}$. That is, $x-x b=x a$, as required.

Now, finite intersections of maximal ideals are evidently open. Conversely, if $I$ is any open ideal then $I$ contains a finite intersection of maximal ideals; consequently, any maximal divisor of $I$ is one of them, because a maximal ideal is prime. Therefore, $\mathrm{Cl} I$ is the intersection of a finite number of maximal divisors of $I$, using I.C.P. But, 
since $I$ is an open subgroup it is closed. Thus the result follows.

Let $\sigma$ denote the family of all ideals $I$ of a ring $R$ satisfying the condition: For every maximal ideal $M$ of $R$ and for every $x \in M$, $(x+I) \cap \pi(M)$ is nonnull. From the previous lemma, $\sigma$ is closed under finite intersection. Thus as a basis of neighbourhoods at zero, $\sigma$ defines a ring topology on $R$.

THEOREM 3. Among the group topologies with I.C.P. on a ring $R$, there is one possessing the largest collection of open ideals-viz. $\sigma$.

Proof. By direct appeal to the definition of $\sigma, \mathrm{Cl} \pi(M)=M$ for every maximal ideal $M$. So, I.C.P. of $\sigma$ follows invoking Theorem 1 . Obviously, any open ideal in whatever group topology with I.C.P. on $R$ belongs to $\sigma$.

REMARK 1. The topology on $R$ given by $\sigma$ is Hausdorff. In fact, the $\sigma$-topology is finer than any group topology with I.C.P. and having an ideal basis of neighbourhoods at zero.

REMARK 2. The following statements are equivalent in a ring $R$.

(i ) $\sigma$ consists of all ideals.

(ii ) $\sigma$-topology is discrete.

(iii) $R$ is a (von Neumann) regular ring.

(iv) $\pi(M)=M$ for every maximal ideal $M$.

The proof is evident by the use of [1, Th. 3.16].

REMARK 3. It does not seem possible to algebraise the pseudocompactness of a topological space so that the results of [2] in this regard may also be generalized.

The following information is in a similar vein to that of Theorem 3. Now of course, the ring need not be semisimple, nor its structure space need be Hausdorff; and the topologies in the ring may well disregard the compatibility criteria.

THEOREM 4. Among the topologies with I.C.P. in a ring $R$, there is one which is the coarsest.

Proof. Define a topology $\tau$ in the ring $R$ by taking all maximal ideals to be a subbase for the closed sets. It suffices to show that $\tau$ has I.C.P. We observe first that an ideal $I$ is contained in the set union of a finite number of prime ideals $P_{r}$ only if it is contained in 
some one $P_{r}$. For, assume without losing generality that any pair of prime ideals in this finite collection are incomparable. Then we may choose elements $x_{r} \in\left(\bigcap_{s \neq r} P_{s}\right)-P_{r}$. If $i_{r} \in I-P_{r}$ would also exist, it will amount to a contradiction viz., $\sum i_{r} x_{r}$ belongs to $I$ but not to any $P_{r}$. Now, the closure of an ideal is the intersection of basic closed sets in $\tau$-which are finite unions of maximal ideals. Therefore, Cl $I$ must be an intersection of maximal ideals. The proof is complete.

The writer thanks Prof. M. Venkataraman for his inspiration and encouragement.

\section{REFERENCES}

1. L. Gillman, Rings with Hausdorff structure space, Fund. Math. 45 (1957), 1-16.

2. L. A. Hinrichs, Open ideals in C(x), Pacific J. Math. 14 (1964), 1255-1263.

Received November 14, 1966.

Madurai University

MADURAI-2, INDIA

AND

Case Western Reserve University 


\section{PACIFIC JOURNAL OF MATHEMATICS}

\section{EDITORS}

\section{H. ROYDEN}

Stanford University

Stanford, California

\section{J. P. Jans}

University of Washington

Seattle, Washington 98105

\section{J. DUGUNDJI}

Department of Mathematics

Rice University

Houston, Texas 77001

RICHARD ARENS

University of California

Los Angeles, California 90024

\section{ASSOCIATE EDITORS}
E. F. BeCKenbaCH
B. H. NeumanN
F. WOLF
K. YosIDA

\section{SUPPORTING INSTITUTIONS}

UNIVERSITY OF BRITISH COLUMBIA CALIFORNIA INSTITUTE OF TECHNOLOGY UNIVERSITY OF CALIFORNIA MONTANA STATE UNIVERSITY UNIVERSITY OF NEVADA NEW MEXICO STATE UNIVERSITY OREGON STATE UNIVERSITY UNIVERSITY OF OREGON OSAKA UNIVERSITY UNIVERSITY OF SOUTHERN CALIFORNIA
STANFORD UNIVERSITY UNIVERSITY OF TOKYO UNIVERSITY OF UTAH WASHINGTON STATE UNIVERSITY UNIVERSITY OF WASHINGTON

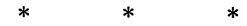

AMERICAN MATHEMATICAL SOCIETY CHEVRON RESEARCH CORPORATION TRW SYSTEMS

NAVAL ORDNANCE TEST STATION 


\section{Pacific Journal of Mathematics}

\section{Vol. 24, No. $1 \quad$ May, 1968}

Harry P. Allen, Lie algebras of type $D_{4}$ over algebraic number fields ...... 1

Charles Ballantine, Products of positive definite matrices. II............ 7

David W. Boyd, The spectral radius of averaging operators ............ 19

William Howard Caldwell, Hypercyclic rings ................... 29

Francis William Carroll, Some properties of sequences, with an application

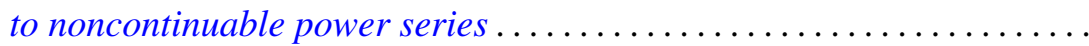

David Fleming Dawson, Matrix summability over certain classes of

sequences ordered with respect to rate of convergence ........... 51

D. W. Dubois, Second note on David Harrison's theory of preprimes. . . . . 57

Edgar Earle Enochs, A note on quasi-Frobenius rings.............. 69

Ronald J. Ensey, Isomorphism invariants for Abelian groups modulo bounded groups ................................ 71

Ronald Owen Fulp, Generalized semigroup kernels ................ 93

Bernard Robert Kripke and Richard Bruce Holmes, Interposition and approximation ................................. 103

Jack W. Macki and James Sai-Wing Wong, Oscillation of solutions to second-order nonlinear differential equations ..................

Lothrop Mittenthal, Operator valued analytic functions and generalizations

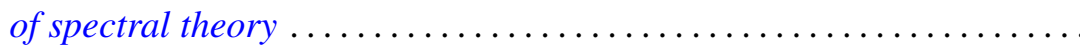

T. S. Motzkin and J. L. Walsh, A persistent local maximum of the pth power deviation on an interval, $p<1 \ldots \ldots \ldots \ldots \ldots \ldots \ldots \ldots \ldots \ldots . \ldots \ldots$

Jerome L. Paul, Sequences of homeomorphisms which converge to homeomorphisms ...........................

Maxwell Alexander Rosenlicht, Liouville's theorem on functions with elementary integrals.

Joseph Goeffrey Rosenstein, Initial segments of degrees .

$\mathrm{H}$. Subramanian, Ideal neighbourhoods in a ring ............

Dalton Tarwater, Galois cohomology of abelian groups . .

James Patrick Williams, Schwarz norms for operators ... .

Raymond Y. T. Wong, A wild Cantor set in the Hilbert cube. 